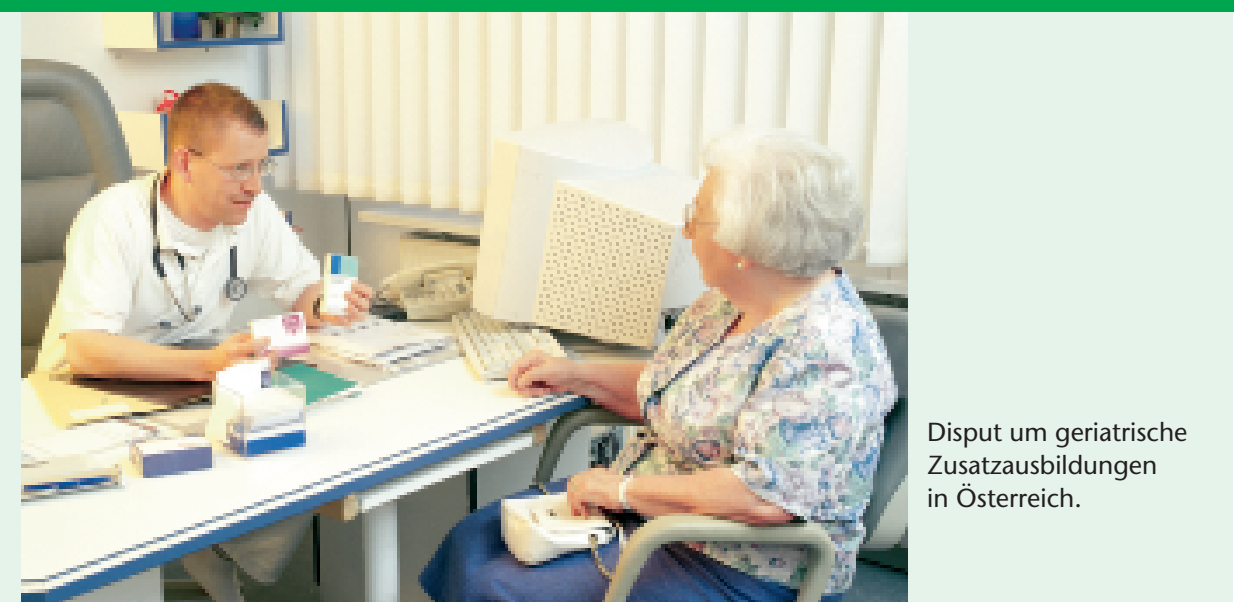

\section{«Ho-Ruck-Aktion» bei Ärzteausbildung}

Als «unkoordinierte Ho-Ruck-Aktion» hat der Präsident der Österreichischen Ärztekammer (ÖÄK), Dr. Reiner Brettenthaler, den Plan des Gesundheitsministeriums bezeichnet, für einzelne medizinische Fächer neue geriatrische Zusatzausbildungen aus dem Boden zu stampfen. Das Vorhaben, dreijährige Spezialausbildungen in Geriatrie für Internisten, Neurologen, Psychiater und physikalische Ärzte im Zuge der Neuregelung der Ärzteausbildungsordnung zu etablieren, sei «nicht mit der Ärztekammer akkordiert, mehr als undurchdacht und uns völlig unverständlich», kritisierte der ÖÄK-Präsident. Durch die angestrebte Regelung würden Allgemeinmediziner und andere ärztliche Fachgruppen mit breitem Erfahrungsschatz in der Versorgung alter Menschen von einer Zusatzqualifikation quasi ausgeschlossen. Er halte diesen Ansatz für «wenig sinnvoll», distanzierte sich Brettenthaler vom Vorhaben des Ministeriums. In der Ärztekammer sei man «mehr als irritiert über diesen politischen Schnellschuss», zumal die von der ÖÄK entwickelte neue Ärzteausbildungsordnung seit weit über einem Jahr im Ministerium zur Unterschrift liege und jetzt quasi in letzter Minute diese «unausgegorene Idee» auf den Tisch komme, erklärte auch der Bildungsreferent der ÖÄK, Dr. Terje Hovdar. Es hätten in dieser Zeit genügend Gesprächsmöglichkeiten bestanden, aber weder die Österreichische Ärztekammer noch die wissenschaftlichen Gesellschaften für Allgemeinmedizin, Innere Medizin, Neurologie, Psychiatrie und Physikalische Medizin seien im Rahmen einer Begutachtung vorweg in die Pläne des Ministeriums eingebunden worden. Dies obgleich man dem Ministerium schon im Vorjahr angeboten habe, zum Thema Geriatrie einen interdisziplinären Konsens im Rahmen der Kammer unter Einbindung aller in der geriatrischen Versorgung etablierten Fächer und ein Strukturkonzept zu erarbeiten. Die Betreuung alter Menschen sei zwar das Thema der Zukunft in der Medizin. Aber dazu müsse man sich interdisziplinär an einen Tisch setzen und Betreuungsszenarien und
Notwendigkeiten zwischen allen in der Altersmedizin tätigen Ärztegruppen und anderen Berufsgruppen durchdiskutieren. Fachvertretungen und wissenschaftliche Gesellschaften lehnten die ministerielle Initiative einheitlich ab. Brettenthaler und Hovdar forderten das Gesundheitsministerium erneut auf, die von der ÖÄK vorgelegte neue Ärzteausbildungsordnung endlich zu unterzeichnen, die seit elf Jahren überfällig sei, und auf den «unausgegorenen Plan dreijähriger Zusatzausbildungen» $\mathrm{zu}$ verzichten

(Österreichische Ärztekammer)

\section{Auch Naturheilverfahren haben Nebenwirkungen}

«Jedem sollte klar sein, dass man auch bei pflanzlichen Arzneistoffen nicht allein mit therapeutischen Wirkungen, sondern auch mit unerwünschten Nebenwirkungen rechnen muss»: Dies sagte Prof. Dr. Ernst-Gerhard Loch, stellvertretender Vorsitzender des Deutschen Senats für ärztliche Fortbildung der Bundesärztekammer anlässlich des 30. Interdisziplinären Forums der Bundesärztekammer. Naturheilverfahren sind so alt wie die Medizin. In Zeiten ökonomischen Drucks auf die medizinische Versorgung neigen Patientinnen und Patienten gerade bei Befindlichkeitsstörungen allerdings verstärkt dazu, sich mit Naturheilmitteln selbst zu therapieren. Auch vor einer therapeutischen Entscheidung mit naturgemässen Methoden muss eine exakte Diagnostik nach den Grundregeln der medizinischen Wissenschaft erfolgen, forderte Loch. Zu den klassischen Naturheilverfahren zählt auch die Lehre von der Ernährung (Diätetik). Gerade ihr kommt zum Beginn des 21. Jahrhunderts eine besondere Bedeutung zu. «Der Einfluss der Ernährung auf Entstehung, Verhütung und Behandlung der modernen Zivilisationsseuchen Arteriosklerose und Diabetes mellitus kann kaum überschätzt werden», sagte Prof. Dr. Karl Huth von der Justus-Liebig-Universität in Giessen. Ärzte müssten sich daher vermehrt der Ernährungsberatung widmen. Die Motivation der Patien- ten für bestimmte Änderungen im Lebensstil und Ernährungsverhalten sei unzulänglich. Insbesondere Überernährung und Fettsucht hätten in jüngster Zeit zugenommen, so dass man heute von einer weltweiten Epidemie sprechen könne. «Laut Weltgesundheitsbericht von 2002 sind eine Milliarde Erwachsene übergewichtig, davon 300 Millionen adipös», erklärte Huth. In südeuropäischen Ländern seien bis zu 30 Prozent der Kinder übergewichtig oder adipös, in Deutschland ca. 20 Prozent. Typische Folgen seien Diabetes mellitus Typ II, Hypertonie oder in späteren Jahren adipositasabhängige Krebsarten wie Darm- oder Prostatakrebs.

Wenn heute mit der Regel «Fünfmal am Tag Obst und Gemüse» zu mehr Kohlenhydraten in der Kost geraten werde, dann sei damit auch ein höherer Ballaststoffverzehr gemeint. Aus der Analyse aktueller Studien könne man schliessen, dass eine ausreichende Zufuhr von Ballaststoffen das Darmkrebsrisiko senken könne. «Statt den heute bei uns üblichen 20 Gramm sollten täglich mehr als 30 Gramm Ballaststoffe pro $1000 \mathrm{kcal}$ genossen werden», empfahl Huth.

(Bundesärztekammer)

\section{Dicke Männer bringen sich seltener um als dünne}

Dicke Männer bringen sich nach einer Studie seltener um als dünne. Zwar hätten übergewichtige Männer viele gesundheitliche Probleme wie hohen Blutdruck, Diabetes und verstopfte Arterien. Aber Depressionen gehörten offenbar nicht dazu, sagte der Wissenschafter Finn Rasmussen vom Karolinska Institut im schwedischen Radio. Dagegen hätten frühere Studien mit Frauen gezeigt, dass die dickeren unter ihnen eher zu Depressionen - und damit auch zum Selbstmord neigten. «Es gibt viele Gründe für Selbstmord, aber die Tatsache, dass übergewichtige Männer weniger in Gefahr sind, stützt die Vermutung, dass es biologische Gründe dafür gibt», sagte Rasmussen. Eine schlüssige Erklärung könnten die Forscher bislang nicht liefern. "Wir glauben, dass es einen zugrundeliegenden biologischen Mechanismus gibt, der dicke Männer schützt, auch wenn sie an gesellschaftlicher Diskriminierung leiden.» Allerdings habe es für Männer keinen Sinn, sich Kilos anzufuttern, um nicht depressiv zu werden, warnte der Wissenschafter - dafür habe das Dicksein zu viele «schlechte Nebenwirkungen». Die Untersuchung beruht auf ärztlichen Untersuchungen der jungen Schweden, die zwischen 1968 und 1999 zum Militär eingezogen worden waren: Es handelt sich dabei um 1,3 Millionen Männer, die bei der Untersuchung 18 oder 19 Jahre alt waren.

$(s d a / d p a)$ 